\title{
Extração Automática de Contornos de Edificações a partir de Dados LiDAR Aerotransportado
}

\author{
Automatic Building Boundary Extraction from Airborne LiDAR Data
}

\author{
André Caceres Carrilho ${ }^{1}$ \\ Renato César dos Santos ${ }^{1}$ \\ Guilherme Gomes Pessoa ${ }^{1}$ \\ Mauricio Galo 1,2
}

Recebido em dezembro de 2018.

Aprovado em setembro de 2019.

\begin{abstract}
RESUMO
Este artigo tem o objetivo de avaliar a qualidade da extração automática de contornos de edificações a partir de dados LiDAR aerotransportado visando verificar se os erros obtidos nas áreas de edificações são compatíveis com os critérios estabelecidos pela Lei 10.406, de 10 de janeiro de 2002. Nesse contexto, duas abordagens são comparadas: uma presente no software LAStools (lasboundary) e um variante do algoritmo a-shape, cujo parâmetro a é estimado de modo adaptativo para cada edificação. As abordagens foram aplicadas em dois conjuntos de dados, um com densidade média de $5,8 \mathrm{pts} / \mathrm{m}^{2}$ e outro com $12,5 \mathrm{pts} / \mathrm{m}^{2}$. A partir dos experimentos foi possível determinar a qualidade obtida em cada caso, considerando diferentes indicadores: F-score, erro relativo em área, e a métrica PoLiS. Os resultados indicam que para a maior parte dos casos ambas abordagens apresentaram acurácia compatível com a Lei mencionada. De modo geral, o método variante do algoritmo a-shape forneceu melhores resultados, quando comparado ao uso da ferramenta lasboundary, na maior parte dos experimentos, indicando que a determinação dinâmica do parâmetro a contribui para a qualidade final da extração, principalmente em edificações mais complexas. Por fim, o uso da nuvem de pontos com maior densidade resultou em maior acurácia na extração de polígonos das edificações.
\end{abstract}

PALAVRAS-CHAVE: Extração de contornos de edificações. Dados LiDAR aerotransportado. $\alpha$-shape.

\footnotetext{
${ }^{1}$ Universidade Estadual Paulista (UNESP) - Faculdade de Ciências e Tecnologia (FCT). Programa de Pós-Graduação em Ciências Cartográficas (PPGCC), Brasil. E-mail(s): andre.carrilho@unesp.br, renato_cstos@hotmail.com, guigpessoa@gmail.com

${ }^{2}$ Universidade Estadual Paulista (UNESP) - Faculdade de Ciências e Tecnologia (FCT). Departamento de Cartografia, Brasil. E-mail: mauricio.galo@unesp.br
} 


\begin{abstract}
:
This paper aims to evaluate the quality of the automatic extraction of building contours from airborne LiDAR data in order to verify if the errors obtained in the building roof areas are compatible with the criteria established by the Brazilian Law $n^{\circ} 10.406$, January 10 $10^{\text {th }}$, 2002. Two approaches were compared: one present in LAStools software (lasboundary) and the other being a variant of the a-shape algorithm, whose parameter a is estimated adaptively for each building. In addition, both approaches were applied to two datasets, one with an average point density of $5.8 \mathrm{pts} / \mathrm{m}^{2}$ and the other with $12.5 \mathrm{pts} / \mathrm{m}^{2}$. In this work three quality parameters were computed: F-score, relative error in area, and PoLiS metric. The assessment of the results indicates that most of the results met the criteria established by the above-mentioned law. The variant of a-shape algorithm seems to be better than the lasboundary, indicating that the dynamic approach to determine the parameter a contributes to the quality, especially on complex buildings. Finally, the use of the point cloud with higher average point density results in higher accuracy in the building boundary extraction.
\end{abstract}

KEYWORDS: Building boundary extraction. Airborne LiDAR data. ashape.

$$
* * *
$$

\title{
Introdução
}

Bases cartográficas atualizadas são importantes para uma série de tarefas, dentre elas: planejamento urbano, monitoramento da expansão urbana e tributação. Além disso, as bases cartográficas servem de subsídio para a composição de Planos Diretores municipais, auxiliando na execução da Política Nacional de Desenvolvimento Urbano, previsto no Estatuto da Cidade, Lei Federal n ${ }^{\circ} 10.257$ (BRASIL, 2001). De acordo com a Constituição Federal (BRASIL, 1988) compete ao município a manutenção do sistema de informações sociais, administrativas, territoriais, cartográficas e outras de interesse, assegurando a sua ampla e periódica divulgação. Entretanto, a criação e a atualização periódica de bases cartográficas são processos que requerem tempo e investimento, tanto em recursos humanos quanto equipamentos e sistemas, que permitem a extração de informações a partir de imagens, ou outras fontes de dados. Nesse sentido, a exploração de dados de sensoriamento remoto e técnicas de extração de feições surge como uma alternativa para a aquisição de informações de interesse. 
Para aplicações urbanas as edificações possuem papel importante, uma vez que recobrem boa parte da superfície. Por tal interesse, o tema relacionado à extração de edificações de forma automática ou semi-automática vem sendo estudado por diversos autores (MACHADO, 2006; KIM e HABIB, 2009; DAL POZ et al., 2009; AWRANGJEB, 2016; CENTENO, DAROS e GARGON, 2016; JUNG, JWA e SOHN, 2017; CARRILHO e GALO, 2018; SANTOS, GALO e CARRILHO, 2018a). Encontram-se na literatura diversas abordagens para a extração de edificações a partir de dados obtidos remotamente. Inicialmente foram propostos métodos baseados em imagens aéreas (MANNO-KOVACS e SZIRANYI, 2015) e/ou imagens obtidas por satélites (WANG et al., 2015). Posteriormente, com o desenvolvimento tecnológico surgiram métodos que utilizam dados LiDAR obtidos por sistemas aerotransportados (GALVANIN e DAL POZ, 2012; KWAK e HABIB, 2014; JUNG, JWA e SOHN, 2017), e nas últimas décadas a comunidade científica tem estudado a utilização conjunta desses dados (KIM e HABIB, 2009; MENDES e DAL POZ, 2013; GHAMISI, HÖFLE e ZHU, 2016; KANG, YANG e ZHONG, 2017; OLIVEIRA e GALO, 2018).

Nesse trabalho os dados LiDAR são considerados como dados de entrada no processo de extração dos contornos das edificações. O termo "dados LiDAR", adotado no decorrer do texto, refere-se ao conjunto de pontos amostrados sobre a superfície terrestre obtido por meio de um Sistema de Varredura a LASER Aerotransportado (SVLA). É relevante destacar que o uso dos dados LiDAR apresenta vantagens e desvantagens em relação aos obtidos por meio de técnicas Fotogramétricas convencionais. As principais vantagens estão relacionadas a aquisição direta da nuvem de pontos tridimensional, o melhor delineamento das faces planas em edificações complexas e a obtenção de maior acurácia na extração de métricas que envolvem altitude, como por exemplo o volume da edificação (GONZÁLESAGUILERA et al., 2013). Entretanto, os dados LiDAR não possuem informação semântica, armazenando apenas a intensidade do pulso. Além 
disso, a qualidade das bordas de edificações e objetos pode ser prejudicada em decorrência do processo de amostragem por SVLA.

A comunidade científica assume como complementares as duas fontes de dados: dados LiDAR e imagens aéreas. Métodos que fazem a utilização conjunta, como os propostos por Machado (2006), Dal Poz (2014), Oliveira (2016), Oliveira e Galo (2018) e Yousefhussien et al. (2018), por exemplo, procuram explorar a potencialidade de cada fonte de dados, isto é, imagens aéreas para delineamento bidimensional dos objetos e dados LiDAR para extrair a informação altimétrica. O problema dessas abordagens acaba tendo cunho prático, uma vez que a obtenção desses dois conjuntos de dados pode encarecer os projetos, o que torna inviável a sua aplicação para uma parcela dos potenciais interessados, como pequenos municípios de países emergentes, por exemplo.

No contexto da extração automática de contornos de edificações a partir de dados LiDAR, diferentes métodos e técnicas podem ser aplicados, dentre os quais pode-se destacar: o algoritmo a-shape (SHEN, 2008; ALBERS, KADA e WICHMANN, 2016; SANTOS et al., 2018b), o algoritmo a-shape adaptativo (SANTOS, GALO e CARRILHO, 2018a), abordagens baseadas na triangulação de Delaunay (MAAS e VOSSELMAN, 1999; AWRANGJEB, 2016), abordagens baseadas no fecho convexo modificado (SAMPATH e SHAN, 2007; LEE et al., 2011; KWAK e HABIB, 2014), o uso da ferramenta lasboundary implementada no software LAStools (ISENBURG, 2016), e o uso de ferramentas disponíveis no software TerraScan (FERREIRA, LAROCCA e CINTRA, 2015), dentre outros.

No trabalho realizado por Ferreira, Larocca e Cintra (2015) os autores realizam a comparação dos resultados obtidos a partir da nuvem de pontos (densidade de $1,5 \mathrm{pts} / \mathrm{m}^{2}$ ) com dados vetoriais adquiridos por técnicas fotogramétricas. $\mathrm{O}$ foco desses autores foi na avaliação da qualidade visando o enquadramento no Padrão de Exatidão Cartográfica dos Produtos Cartográficos Digitais (PEC-PCD) segundo o que estabelece a ET-ADGV (DSG, 2016). 
Considerando a extração automática de contornos de edificações a partir de dados LiDAR, este trabalho busca avaliar a qualidade da extração considerando duas abordagens: (i) ferramenta lasboundary, presente no software LAStools; e (ii) algoritmo a-shape adaptativo. Além de análises comparativas entre os métodos, foram realizadas análises com o intuito de verificar a possibilidade do uso dessas abordagens para o cálculo de áreas de edificações, seguindo os requisitos de qualidade da Lei 10.406, de 10 de janeiro de 2002, que em seu Artigo 500, $\S 1^{\circ}$, dá uma referência ao erro tolerável em área (um vigésimo da área, o que corresponde a 5\%), no caso de ações de compra e venda de imóveis.

\section{Extração automática de contornos de edificações}

A extração automática dos contornos de edificações a partir de dados LiDAR aerotransportado requer alguns processamentos iniciais, dentre eles filtragem e classificação dos pontos. A filtragem tem por objetivo a eliminação de pontos acima do terreno, podendo ser utilizada a fim de rotular os pontos em duas classes: terreno e não terreno. Já o processo de classificação visa rotular os pontos atribuindo informação semântica. Nesse trabalho essas etapas foram realizadas considerando ferramentas disponíveis no software LAStools (ISENBURG, 2016). A filtragem e a classificação foram realizadas a partir das ferramentas lasground e lasclassify, respectivamente. Deste modo, após este pré-processamento pode-se separar os pontos de interesse, isto é, os pontos de edificações, após a remoção dos pontos de chão, vegetação, entre outros. Além disso, é necessário efetuar o agrupamento dos pontos pertencentes a cada uma das edificações, por crescimento de regiões, para em

seguida serem aplicadas as abordagens de extração de contornos de edificações analisadas. 
1.1 Segmentação por crescimento de regiões

O processo de crescimento de regiões proposto por Sampath e Shan (2007) foi adaptado para o caso 3D e aplicado considerando os pontos de edificações irregularmente espaçados como dados de entrada. Para separar os conjuntos de pontos relacionados às diferentes edificações, dois limiares foram adotados, como descrito em Santos et al. (2019a): limiar de distância planimétrica $\left(L_{D x y}\right)$ e altimétrica $\left(L_{D h}\right)$.

A implementação do método de segmentação foi realizada em linguagem de programação $C++$, utilizando o ambiente de desenvolvimento integrado Code::Blocks. Na sequência são apresentados os passos envolvidos no processo de segmentação:

Passo 1: Armazenar os pontos de telhado no vetor $\boldsymbol{V}^{\boldsymbol{T}}=\left[P_{1} P_{2} \ldots P_{i}\right]$;

Passo 2: Selecionar um ponto do vetor $\boldsymbol{V}$ como semente e movê-lo para um vetor $\boldsymbol{B}$, que contém os pontos de uma certa edificação;

Passo 3: Considerando os pontos de $\boldsymbol{V}$, verificar quais pontos satisfazem simultaneamente os seguintes critérios (Equações 1 e 2) (SANTOS et al., 2019a):

$$
\begin{gathered}
\sqrt{\left(x_{i}-x_{j}\right)^{2}+\left(y_{i}-y_{j}\right)^{2}}<L_{D x y} \\
\left|z_{i}-z_{j}\right|<L_{D h}
\end{gathered}
$$

sendo: $p_{i}=\left(x_{i}, y_{i}, z_{i}\right)$ o i-ésimo ponto armazenado no vetor $\boldsymbol{V}$, enquanto $p_{j}=\left(x_{j}, y_{j}, z_{j}\right)$ refere-se as coordenadas do ponto armazenado em $\boldsymbol{B}$;

Passo 4: Os pontos armazenados em $\boldsymbol{V}$ e que atendem os dois critérios são movidos para $\boldsymbol{B}$. Se nenhum ponto for adicionado ao vetor $\boldsymbol{B}$, seguir para o passo 5. Caso contrário, retorna-se ao passo 3;

Passo 5: Gerar um arquivo de saída com os pontos armazenados no vetor $\boldsymbol{B}$;

Passo 6: Os pontos armazenados no vetor $\boldsymbol{B}$ são excluídos. Caso exista algum ponto no vetor $\boldsymbol{V}$, retorna-se ao passo 2. Caso contrário, o processo é finalizado. 
1.2 Extração de contornos com a ferramenta lasboundary

A ferramenta lasboundary do software LAStools pode ser utilizada para obter polígonos a partir das nuvens de pontos, como discutido em Carrilho et al. (2018). Por padrão ela calcula o polígono côncavo formado pelo conjunto de pontos, mas pode ser utilizada também para a determinação de polígonos internos, sendo que o nível de detalhe do polígono pode ser modificado por meio do parâmetro de concavidade ("concavity"), similarmente ao parâmetro a do algoritmo a-shape. Neste trabalho foram utilizadas as opções "disjoint" e "holes", para possibilitar a extração de segmentos desconexos e segmentos internos, respectivamente. Além disso, dois valores para o parâmetro "concavity" foram testados.

\subsection{Extração de contornos usando o algoritmo a-shape}

O algoritmo a-shape consiste na seleção dos segmentos de reta que compõem o contorno de um dado polígono. Como dados de entrada têm-se: o conjunto de dados no espaço $2 \mathrm{D}$ e o parâmetro a (EDELSBRUNNER, KIRKPATRICK e SEIDEL,1983). Segundo Shen (2008) o algoritmo a-shape foi inicialmente desenvolvido pela comunidade ligada a Computação Gráfica e posteriormente aplicado no processamento de dados LiDAR devido a algumas vantagens, tais como: velocidade de processamento, alta acurácia e capacidade de detectar polígonos côncavos e convexos, bem como contornos internos e externos. O desafio no uso desse algoritmo está relacionado à seleção de um valor ótimo para o parâmetro $\alpha$, uma vez que esse depende da densidade de pontos e do nível de detalhamento desejado para os contornos (SANTOS, GALO e CARRILHO, 2019b). Nesse trabalho, o conjunto de pontos corresponde aos pontos LiDAR amostrados sobre as edificações, e o valor do parâmetro a é estimado para cada edificação, utilizando a abordagem apresentada por Santos, Galo e Carrilho (2018a). 
O princípio do algoritmo a-shape é apresentado na Figura 1. Assumindo dois pontos $p_{i}$ e $p_{j}$, e considerando o parâmetro a como sendo o raio do círculo passante pelos pontos $p_{i}$ e $p_{j}$, é possível calcular dois círculos, como ilustrado na Figura 1. O critério estabelecido no algoritmo a-shape consiste em: se não existir nenhum ponto no interior de pelo menos um dos círculos, os pontos $p_{i}$ e $p_{j}$ são marcados como pontos de borda e podem ser conectados para obter um segmento do contorno (Figura 1a). Caso contrário, os pontos $p_{i}$ e $p_{j}$ não formam um segmento do contorno (Figura 1b).

Na Figura 2 são apresentados alguns exemplos considerando diferentes valores de a. Quando a assume um valor ideal é possível obter todos os segmentos de retas do contorno, inclusive o contorno interno (Figura 2a). No caso em que o valor de a tende ao infinito $(\alpha \rightarrow+\infty)$, o resultado coincide com o fecho convexo (Figura 2b). Em contrapartida, quando o valor a tende a zero $(\alpha \rightarrow 0)$, todos os pontos são considerados como pontos de contorno, ou seja, o resultado final coincide com o conjunto de pontos original (Figura 2c).

Figura 1 - Princípio do algoritmo a-shape mostrando em (a) dois pontos de borda e em (b) dois pontos internos

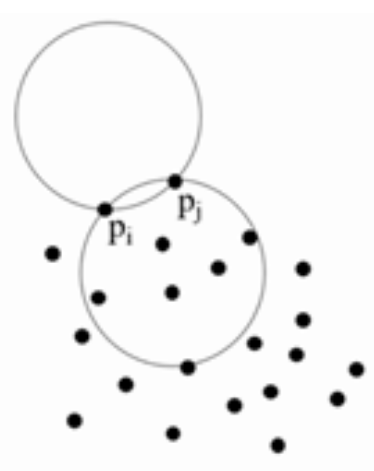

(a)

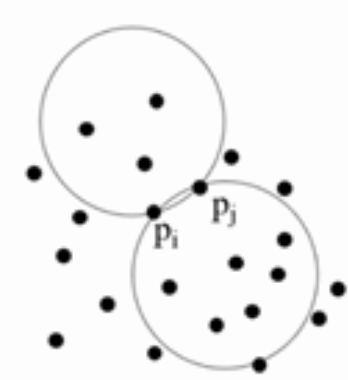

(b)

Fonte: Adaptado de Shen et al. (2011). 
Figura 2 - Resultados obtidos considerando diferentes valores de a

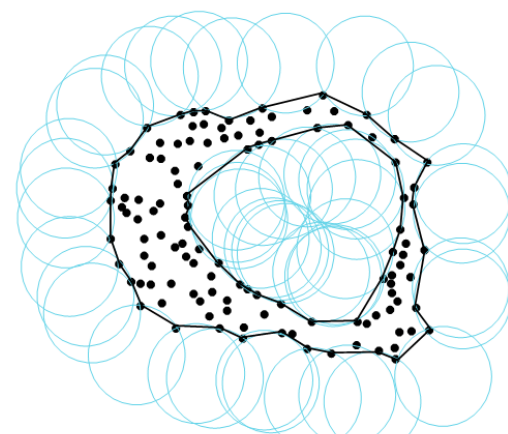

(a)

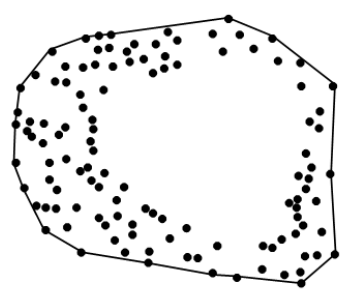

(b)

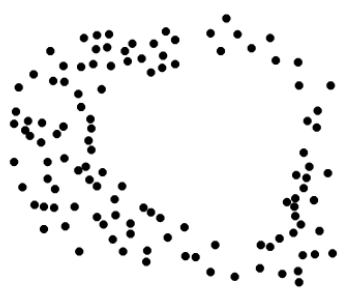

(c)

Fonte: Elaborada pelo autor.

\section{Materiais}

Nos experimentos foram utilizados dados LiDAR do município de Presidente Prudente/SP, os quais pertencem ao Unesp Photogrammetric Data Set, tendo sido adquiridos pela empresa Sensormap Geotecnologia. Além da aquisição de nuvens de pontos por SVLA foram obtidas imagens aéreas de alta resolução e seus respectivos parâmetros de orientação exterior (POE). De acordo com Tommaselli et al. (2018), o sistema de varredura a LASER utilizado foi o RIEGL modelo LMS Q680i. Nesse trabalho foram utilizados dois conjuntos de dados adquiridos a partir de voos com alturas diferentes: $900 \mathrm{~m}$ e $550 \mathrm{~m}$, resultando nas seguintes densidades médias: $5,8 \mathrm{pts} / \mathrm{m}^{2}$ e $12,5 \mathrm{pts} / \mathrm{m}^{2}$, respectivamente (Figura $3 \mathrm{a}$ e $3 \mathrm{~b}$ ). $\mathrm{O}$ artigo mencionado reporta que, após a fototriangulação, o Erro Médio Quadrático (EMQ) determinado a partir de doze pontos de verificação foi da ordem de $4 \mathrm{~cm}$ (em planimetria) e $7 \mathrm{~cm}$ (em altimetria). 
Figura 3 - Detalhes de uma área urbana onde podem ser vistas as nuvens de pontos obtidas por SVLA com diferentes alturas de voo e densidades

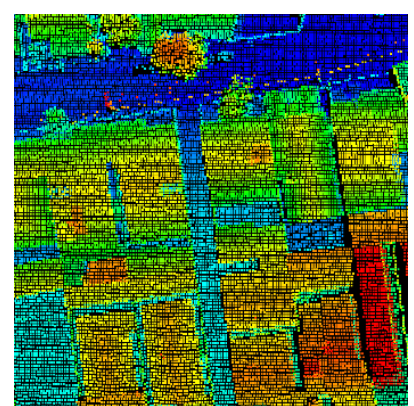

(a) $900 \mathrm{~m}-5,8 \mathrm{pts} / \mathrm{m}^{2}$

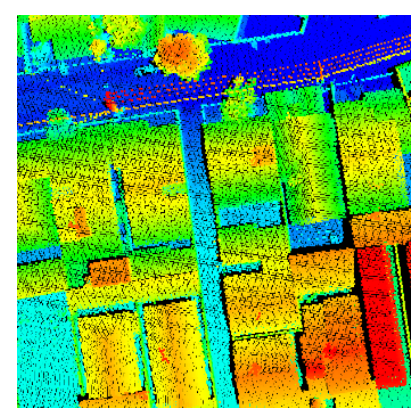

(b) $550 \mathrm{~m}-12,5 \mathrm{pts} / \mathrm{m}^{2}$

Fonte: Elaborada pelo autor.

Os polígonos utilizados como referência para a avaliação quantitativa dos contornos extraídos foram obtidos manualmente a partir de restituição fotogramétrica das imagens aéreas disponíveis no conjunto de dados, utilizando o software ERDAS IMAGINE Photogrammetry 2015 (anteriormente conhecido por Leica Photogrammetry Suite - LPS) com o módulo PRO600 do MicroStation. As imagens aéreas utilizadas possuem um Ground Sample Distance (GSD) da ordem de $8 \mathrm{~cm}$. O algoritmo a-shape e as métricas usadas na análise quantitativa foram implementados na linguagem $\mathrm{C}++$. Em alguns processamentos, além do software LAStools foi utilizado o software livre CloudCompare (versão 2.8: http://www.cloudcompare.org) tanto para a preparação quanto visualização dos dados e resultados.

\section{Experimentos realizados e resultados}

Para a realização dos experimentos foram selecionadas quatro edificações cujos contornos apresentam diferentes características geométricas, como pode ser visto na Figura 4. A primeira edificação (E1) possui uma forma simples, assemelhando-se a um retângulo. A segunda edificação (E2) possui uma maior complexidade, sendo formada por segmentos côncavos e convexos. A edificação E2 possui uma fenda no lado esquerdo, como pode ser visto a partir do perfil da componente altimétrica. A 
terceira edificação (E3) também é uma edificação complexa, a qual é composta por um contorno interno correspondente a um jardim de inverno. A última edificação (E4) possui formato de "H", caracterizando a presença de segmentos côncavos.

Figura 4 - Edificações consideradas nos experimentos (figuras em escalas diferentes), e perfil da altura correspondente a edificação E2
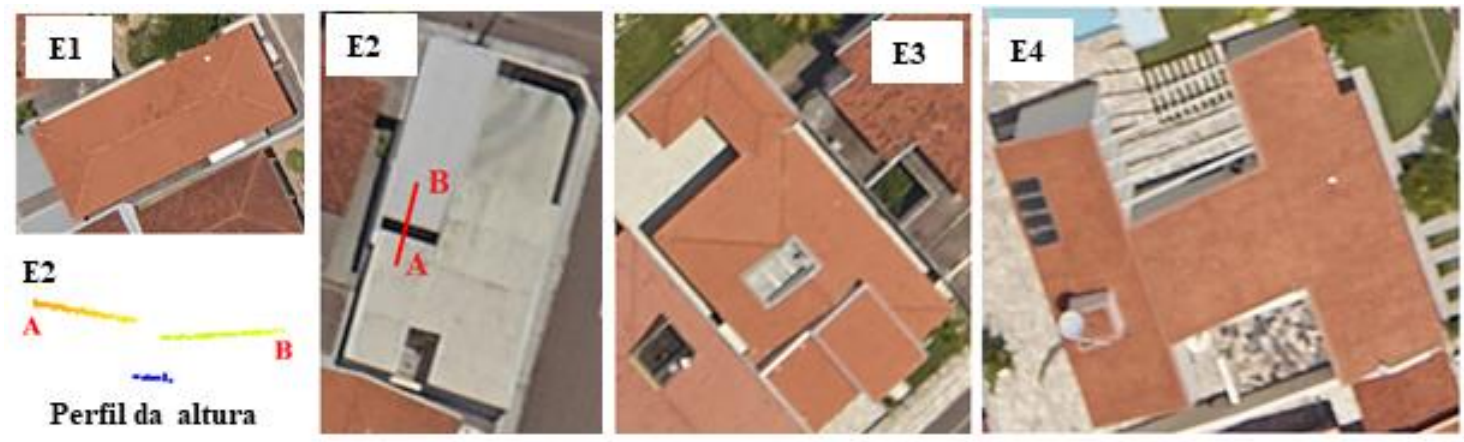

Fonte: Elaborada pelo autor.

Os contornos das edificações foram extraídos a partir dos dados LiDAR usando a ferramenta lasboundary do software LAStools e o algoritmo ashape. No primeiro caso os resultados foram gerados considerando dois valores diferentes para o parâmetro de concavidade: $c=1 \mathrm{~m}$ e $c=2 \mathrm{~m}$. Já no algoritmo $a$-shape o parâmetro a foi determinado de forma adaptativa para cada edificação (SANTOS, GALO e CARRILHO, 2018a, 2019b).

$\mathrm{Na}$ Figura 5 são apresentados os contornos extraídos para as edificações selecionadas (E1-E4), os quais foram obtidos a partir do conjunto de pontos com densidade média de $5,8 \mathrm{pts} / \mathrm{m}^{2}$. A Figura 6 mostra os contornos correspondentes às edificações E1 e E4, os quais foram extraídos sobre o conjunto com maior densidade $\left(12,5 \mathrm{pts} / \mathrm{m}^{2}\right)$. Nas Figuras 5 e 6 , a primeira e segunda coluna apresentam os resultados obtidos por meio da ferramenta lasboundary usando os parâmetros de concavidade iguais a $1 \mathrm{~m}$ e $2 \mathrm{~m}$, respectivamente; enquanto que na terceira coluna são mostrados os resultados referentes ao algoritmo $\alpha$-shape. 
Figura 5 - Contornos de edificações extraídos sobre o conjunto de dados com $5,8 \mathrm{pts} / \mathrm{m}^{2}$.

Resultados usando a ferramenta lasboundary ( $c=1 \mathrm{~m}$ e $c=2 \mathrm{~m}$ ) e o algoritmo a-shape adaptativo

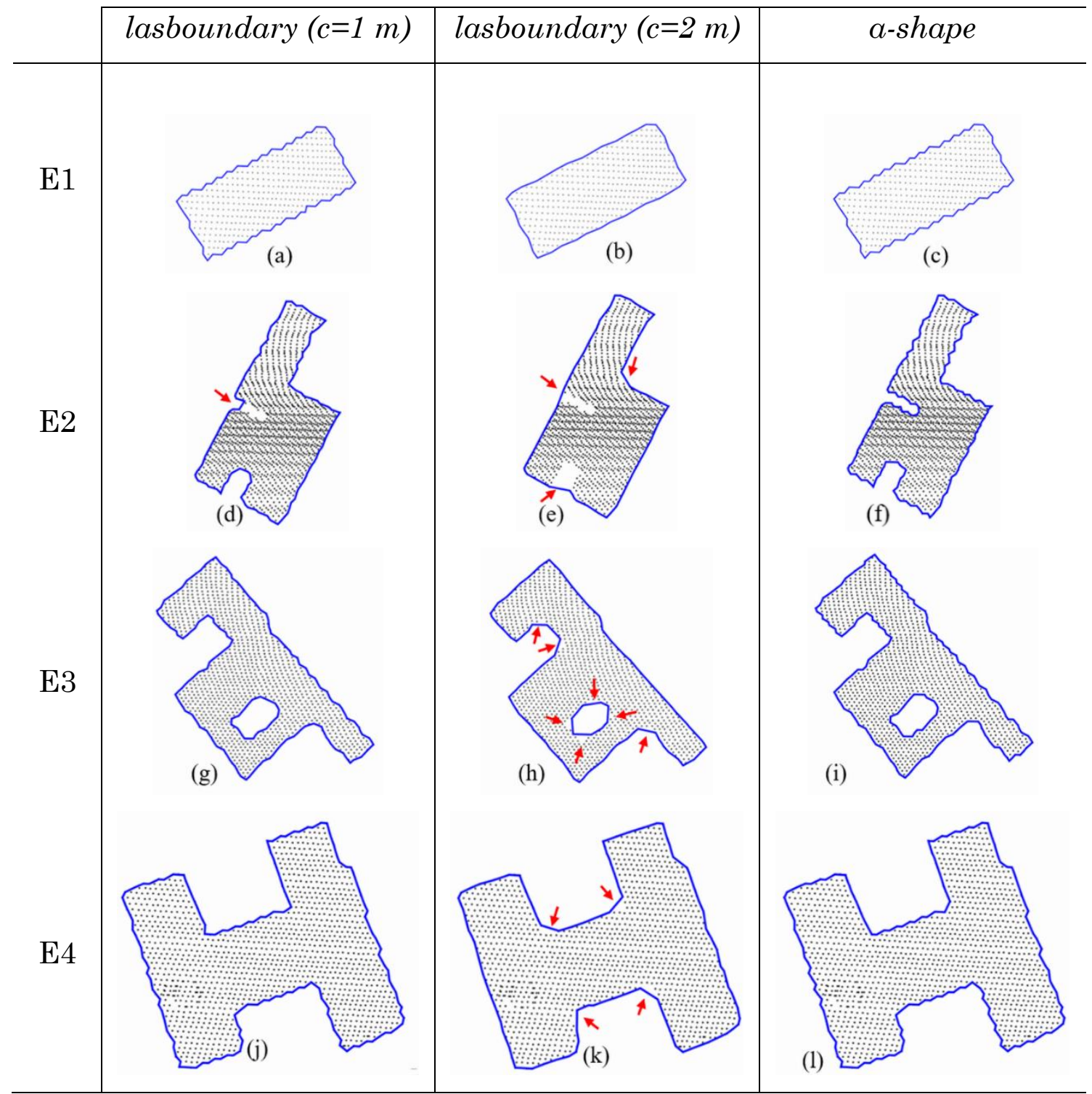

Fonte: Elaborada pelo autor.

Visando realizar a análise quantitativa, os seguintes parâmetros de qualidade foram considerados: $F$-score (SOKOLOVA, JAPKOWICZ e SZPAKOWICZ, 2006; CARRILHO, IVANOVÁ e GALO, 2017), a distância PoLiS (AVBELJ, MÜLLER e BAMLER, 2015), e o erro relativo em área (ER), obtido a partir de $E_{R}=100\left(A_{E}-A_{R}\right) / A_{R}$, onde $A_{E}$ corresponde a área obtida pelo método de extração analisado e $A_{R}$ a área de referência. $O$ erro relativo 
em área foi calculado com o intuito de verificar se os erros obtidos atendem ao que preconiza a Lei 10.406, de 10 de janeiro de 2002, que em seu Artigo 500, $\S 1^{\circ}$, dá uma referência ao erro tolerável em área (um vigésimo da área, o que corresponde a 5\%), para o caso de ações de compra e venda de imóveis.

Figura 6 - Contornos de edificações extraídos sobre o conjunto de dados com 12,5 pts $/ \mathrm{m}^{2}$.

Resultados usando a ferramenta lasboundary ( $c=1 \mathrm{~m}$ e $c=2 \mathrm{~m}$ ) e o algoritmo a-shape adaptativo

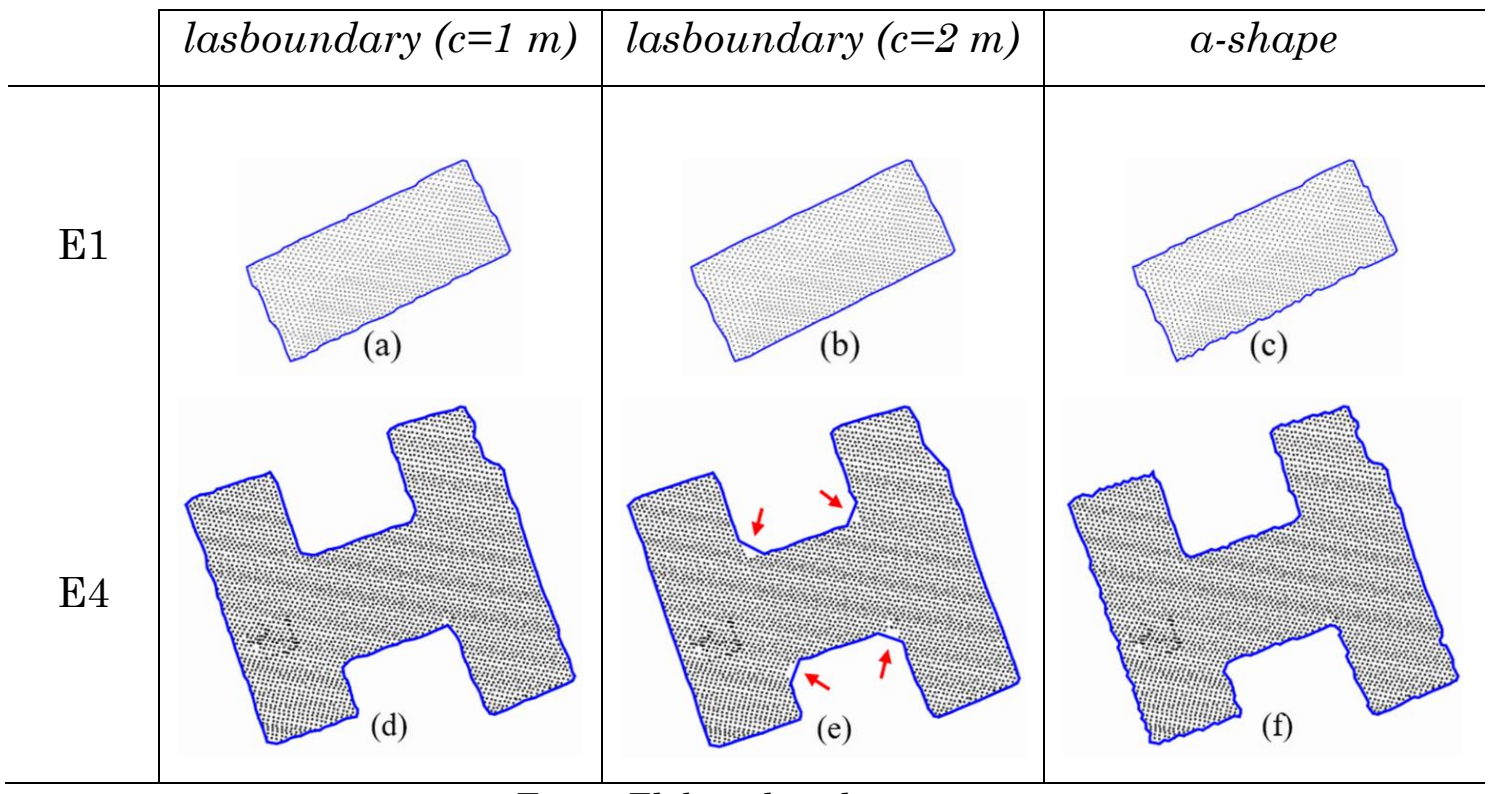

Fonte: Elaborada pelo autor.

Como mencionado anteriormente (Seção 2), os contornos de referência foram obtidos por meio de restituição fotogramétrica. Na prática, os sistemas de varredura a LASER possibilitam, em geral, uma maior acurácia vertical com relação à interseç̧ão fotogramétrica. Isso ocorre principalmente devido à qualidade da medida do tempo de propagação e consequente qualidade da distância sensor-alvo obtida pelo SVLA e pelo fato da qualidade da solução da interseç̧ão dos raios homólogos ser dependente da qualidade do estabelecimento da correspondência (matching) bem como da relação entre a aerobase e a altura de voo, que pode ocasionar uma condição geométrica não favorável à determinação da componente vertical. Entretanto, como neste trabalho a comparação está sendo feita a partir da área da projeção ortogonal 
da edificação no plano, apenas as componentes planimétricas são utilizadas no cálculo das áreas e dos parâmetros de qualidade.

A Tabela 1 apresenta os valores em área relacionados aos polígonos de referência, e aos polígonos extraídos, bem como os parâmetros de qualidade para as diferentes estratégias e densidades de pontos $\left(5,8 \mathrm{pts} / \mathrm{m}^{2}\right.$ e $12,5 \mathrm{pts} / \mathrm{m}^{2}$ ). Nesta tabela também são mostrados os valores dos parâmetros utilizados relativos à concavidade, bem como o valor de a estimado para cada edificação. Para cada edificação são destacados os melhores valores.

Tabela 1 - Parâmetros de qualidade para diferentes edificações, estratégias e densidade de pontos ( $\mathrm{A}_{R}$ - Área de Referência; $\mathrm{A}_{\mathrm{E}}$ - Área de Extraída; e $\mathrm{E}_{\mathrm{R}}$ - Erro Relativo)

\begin{tabular}{|c|c|c|c|c|c|c|c|}
\hline & ID & Estratégia & $\begin{array}{l}\mathrm{A}_{\mathrm{R}} \\
\left(\mathrm{m}^{2}\right)\end{array}$ & $\begin{array}{c}A_{E} \\
\left(m^{2}\right)\end{array}$ & $\begin{array}{l}E_{R} \\
(\%)\end{array}$ & $\begin{array}{c}\text { F-score } \\
(\%)\end{array}$ & $\begin{array}{c}\text { PoLiS } \\
\text { (m) }\end{array}$ \\
\hline \multirow{12}{*}{ 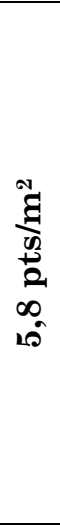 } & \multirow{3}{*}{ E1 } & lasboundary $(c=1 \mathrm{~m})$ & \multirow{3}{*}{164,47} & 157,23 & $-4,40$ & 97,06 & 0,264 \\
\hline & & lasboundary $(c=2 \mathrm{~m})$ & & 164,97 & 0,30 & 98,12 & 0,227 \\
\hline & & $\alpha$-shape $(\alpha=0,75 \mathrm{~m})$ & & 157,45 & $-4,27$ & 97,12 & 0,255 \\
\hline & \multirow{3}{*}{ E2 } & lasboundary $(c=1 \mathrm{~m})$ & \multirow{3}{*}{131,89} & 133,30 & 1,07 & 96,37 & 0,377 \\
\hline & & lasboundary $(c=2 \mathrm{~m})$ & & 142,23 & 7,84 & 94,72 & 0,544 \\
\hline & & $\alpha$-shape $(\alpha=0,37 \mathrm{~m})$ & & 127,88 & $-3,04$ & 96,54 & 0,146 \\
\hline & \multirow{3}{*}{ E3 } & lasboundary $(c=1 \mathrm{~m})$ & \multirow{3}{*}{203,46} & 198,26 & $-2,56$ & 96,52 & 0,200 \\
\hline & & lasboundary $(c=2 \mathrm{~m})$ & & 207,43 & 1,95 & 94,27 & 0,302 \\
\hline & & $\alpha$-shape $(\alpha=0,51 \mathrm{~m})$ & & 196,39 & $-3,47$ & 96,22 & 0,178 \\
\hline & \multirow{3}{*}{$\mathrm{E} 4$} & lasboundary $(c=1 \mathrm{~m})$ & \multirow{3}{*}{300,03} & 296,66 & $-1,12$ & 97,30 & 0,219 \\
\hline & & lasboundary $(c=2 \mathrm{~m})$ & & 306,11 & 2,03 & 97,15 & 0,304 \\
\hline & & $\alpha-$ shape $(\alpha=0,55 \mathrm{~m})$ & & 296,78 & $-1,08$ & 97,26 & 0,217 \\
\hline \multirow{6}{*}{ 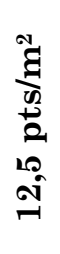 } & \multirow{3}{*}{ E1 } & lasboundary $(c=1 \mathrm{~m})$ & \multirow{3}{*}{164,47} & 166,80 & 1,42 & 97,82 & 0,167 \\
\hline & & lasboundary $(c=2 \mathrm{~m})$ & & 168,80 & 2,63 & 97,65 & 0,168 \\
\hline & & $\alpha$-shape $(\alpha=0,40 \mathrm{~m})$ & & 165,66 & 0,72 & 98,03 & 0,133 \\
\hline & \multirow{3}{*}{$\mathrm{E} 4$} & lasboundary $(c=1 \mathrm{~m})$ & \multirow{3}{*}{300,03} & 302,63 & 0,87 & 98,05 & 0,174 \\
\hline & & lasboundary $(c=2 \mathrm{~m})$ & & 307,45 & 2,47 & 97,77 & 0,243 \\
\hline & & $\alpha$-shape $(\alpha=0,46 \mathrm{~m})$ & & 299,54 & $-0,16$ & 98,09 & 0,154 \\
\hline
\end{tabular}

Fonte: Elaborada pelo autor.

Devido à complexidade do arranjo urbano, algumas edificações podem apresentar áreas de oclusão, por conta da presença de vegetação contigua aos telhados. Visando analisar as abordagens de extração para estas situações também foram consideradas edificações parcialmente oclusas (Figura 7). Nesse caso foi considerado apenas o conjunto de dados com densidade média de $12,5 \mathrm{pts} / \mathrm{m}^{2}$. 
Figura 7 - Contornos de edificações parcialmente oclusas por vegetação (conjunto de dados com 12,5 pts/m²); (a) imagem aérea das edificações; (b) e (c) resultados considerando a ferramenta lasboundary $c=1 \mathrm{~m}$ e $c=2 \mathrm{~m}$; (d) resultados considerando a-shape adaptativo

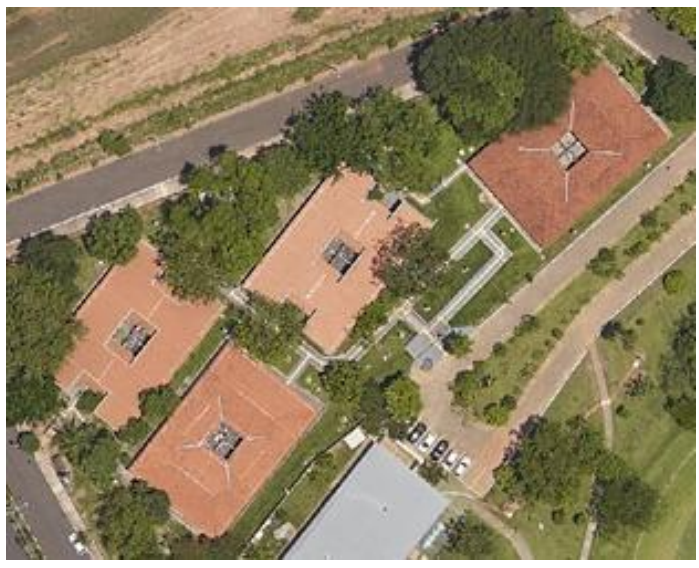

(a)

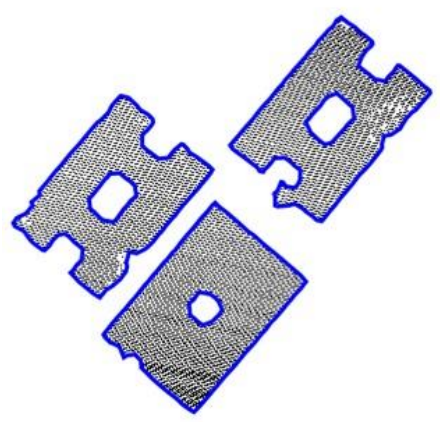

(c)

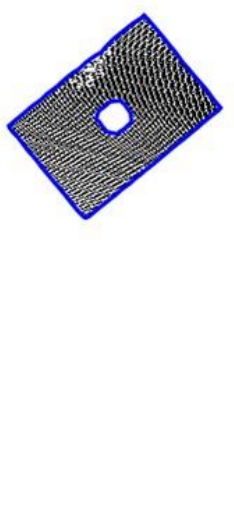

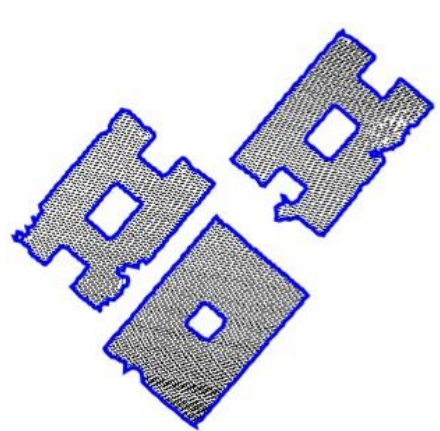

(b)
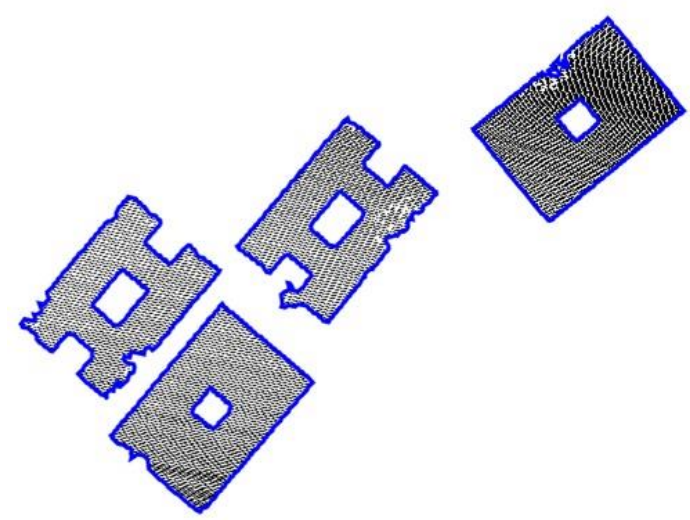

Fonte: Elaborada pelo autor.

Embora o foco desse artigo seja na comparação dos métodos mencionados e não nos tempos de processamentos e no grau de otimização dos algoritmos, é relevante fazer algumas considerações em relação ao tempo de processamento. O tempo de processamento para a aplicação do algoritmo $\alpha$ shape é dependente da triangulação de Delaunay, que é a construção da base da topologia da nuvem de pontos. No pior caso, a triangulação de Delaunay tem complexidade $O\left(n^{2}\right)$. Portanto, o tempo de processamento deve aumentar quadraticamente com o aumento do número de pontos da nuvem. 
Deste modo, considerando um computador com processador Intel Core i7-5500U 2.4 GHz, 8 GB de memória RAM, 500 GB de disco, o processamento da etapa de extração de contornos aplicado à nuvem de pontos correspondente a área da Figura 7 (com quatro edificações), com aproximadamente 17 mil pontos, foi realizado em torno de 12,7 segundos.

\section{Análise dos resultados}

O algoritmo a-shape com parâmetro adaptativo se mostrou eficiente na extração de detalhes e reentrâncias que muitas vezes são negligenciadas pela ferramenta lasboundary, como destacado nas Figuras 5d e 5e. De modo geral, esta ferramenta tende a extrair bordas menos sinuosas, cujo grau de suavidade é diretamente relacionado ao valor do parâmetro utilizado. Analisando a primeira e a segunda colunas das Figuras 5 e 6 é possível verificar que as bordas extraídas pela ferramenta lasboundary com concavidade $1 \mathrm{~m}$ são menos suaves que as extraídas quando considerada concavidade $2 \mathrm{~m}$, a qual extraiu contornos com chanfros em quinas de regiões côncavas, como destacado nas Figuras 5e, 5h, 5k, e $6 \mathrm{e}$.

Devido ao procedimento de amostragem de pontos realizada pelos SVLA, que não necessariamente amostram pontos exatamente sobre as bordas das edificações, espera-se que o valor da área extraída a partir de dados LiDAR seja inferior à de referência, ou seja, que as áreas sejam subestimadas. Esse comportamento foi observado para a maioria dos contornos extraídos a partir do algoritmo a-shape (Tabela 1). Em contrapartida, a maioria dos contornos obtidos pela ferramenta lasboundary apresentaram valor de área superior ao de referência. Essa tendência está diretamente relacionada com a dificuldade de extrair os segmentos côncavos.

Comparando o erro relativo em área $\left(\mathrm{E}_{\mathrm{R}}\right)$ para as edificações $\mathrm{E} 1$ e E4, nas duas densidades $\left(5,8 \mathrm{pts} / \mathrm{m}^{2}\right.$ e $\left.12,5 \mathrm{pts} / \mathrm{m}^{2}\right)$, observa-se que em geral os resultados melhoram com o aumento da densidade, com exceção do processamento realizado usando o lasboundary com concavidade $2 \mathrm{~m}$, no qual 
o erro aumentou. Além disso, ao aumentar a densidade de pontos o algoritmo a-shape resultou em uma taxa de melhora maior do que o lasboundary quando considerado com concavidade $1 \mathrm{~m}$. Ainda tratando do erro relativo em área, dos 18 erros relativos mostrados na Tabela 1, apenas um deles não atendeu a legislação, ou seja, 94,4 \% das áreas calculadas atenderam ao critério estabelecido pela Lei 10.406, de 10 de janeiro de 2002, isto é, de apresentar uma diferença máxima de 5\% (BRASIL, 2002). A única exceção foi para a edificação E2, cujo polígono extraído com o lasboundary e parâmetro de concavidade $c=2 \mathrm{~m}$ resultou em um erro relativo em área de 7,8\%.

Apesar do erro relativo em área ser considerado pela Lei 10.406, alguns erros podem ser omitidos por tal métrica. No caso da edificação E2, a estratégia usando a ferramenta lasboundary $(c=1)$ apresentou o menor valor de erro relativo em área. No entanto, nota-se que a fenda do lado esquerdo da edificação não foi representada (Figura 5d), sendo o melhor resultado obtido pelo algoritmo a-shape (Figura 5f). Nesse caso, as métricas F-score e PoLiS mostram-se mais robustas, indicando a necessidade do uso de diferentes métricas na avaliação da qualidade.

Com base nos valores para a métrica $F$-score (Tabela 1 ), verifica-se que os melhores resultados foram obtidos para o método a-shape, com exceção das edificações E1 e E4 extraídas a partir do conjunto de pontos com densidade $5,8 \mathrm{pts} / \mathrm{m}^{2}$. Considerando todas as estratégias, o valor médio da métrica $F$ score foi de aproximadamente $97 \%$, indicando assim uma alta sobreposição entre os contornos extraídos automaticamente daqueles obtidos manualmente e usados como referência.

Analisando os valores obtidos a partir da métrica PoLiS, nota-se que o método variante do algoritmo a-shape apresentou melhores resultados para todas as edificações do conjunto de pontos com densidade $5,8 \mathrm{pts} / \mathrm{m}^{2}$, exceto para a edificação E1. Para o conjunto com densidade $12,5 \mathrm{pts} / \mathrm{m}^{2}$, o algoritmo a-shape também proporcionou os melhores resultados para esta métrica. Esses resultados indicam que o algoritmo a-shape é capaz de produzir contornos com maior acurácia posicional em algumas situações. 
A partir de análise visual da Figura 7, é possível verificar que os contornos extraídos nas regiões oclusas por vegetação não correspondem à realidade. Esse problema está relacionado com a variação significativa da densidade de pontos, quando comparamos a região oclusa e as demais regiões de uma mesma edificação. Ainda assim, vale a pena destacar que a abordagem que considerou a ferramenta lasboundary ( $\operatorname{com} c=2 \mathrm{~m})$, se mostrou menos suscetível às variações de densidade, fato que pode ser justificado pela tendência desta abordagem em negligenciar os detalhes das edificações. Os resultados para as abordagens lasboundary $(\operatorname{com} c=1 \mathrm{~m})$ e a-shape adaptativo foram similares, apresentando uma maior dificuldade na extração dos contornos nas regiões de oclusão.

\section{Conclusões}

Neste trabalho realizou-se a avaliação da extração automática de contornos de edificações a partir de dados LiDAR considerando duas abordagens: aplicação da ferramenta lasboundary, e o uso de um variante do algoritmo a-shape. Por meio de análises visuais e quantitativas observou-se que os resultados são promissores, tendo atendido aos critérios estabelecidos na Lei 10.406 de 10 de janeiro de 2002, principalmente quando utilizado o método variante do algoritmo a-shape. Além disso, o aumento da densidade da nuvem de pontos acarretou em melhorias na extração dos contornos das edificações.

Existem situações em que ambos os métodos podem gerar resultados menos satisfatórios, tais como mudanças abruptas de densidades no contexto de uma edificação. Este problema pode aparecer para edificações que estejam em regiões de sobreposição entre faixas LiDAR, ou que sofrem oclusão por vegetação, por exemplo, onde a densidade de pontos deixa de ser uniforme no interior de uma edificação. Como sugestões para trabalhos futuros sugere-se: avaliar a utilização do algoritmo implementado na ferramenta lasboundary mas estimando o parâmetro concavidade de forma adaptativa com base na 
densidade de pontos, assim como foi feito para o variante do algoritmo ashape; o uso de estratégias específicas para edificações com a presença de áreas de oclusão; e a realização das análises para um conjunto maior de amostras.

\section{Agradecimentos}

Os autores agradecem ao PPGCC - Programa de Pós-Graduação em Ciências Cartográficas da UNESP, à Fundação de Amparo à Pesquisa do Estado de São Paulo - FAPESP (processos: 2016/12167-5 e 2019/05268-8), ao Conselho Nacional de Desenvolvimento Científico e Tecnológico - CNPq (processo 304189/2016-2), e à Coordenação de Aperfeiçoamento de Pessoal de Nível Superior - CAPES (processos: 1582074 e 1692840). Por fim, os autores gostariam de agradecer aos comentários e sugestões realizadas pelos revisores anônimos.

\section{Contribuição dos autores}

O presente artigo culminou de estudos realizados durante o curso de doutorado dos autores André Caceres Carrilho, Renato César dos Santos e Guilherme Gomes Pessoa. O Prof. Dr. Mauricio Galo é orientador dos dois primeiros autores e coorientador do terceiro. As implementações, preparo dos dados e processamentos foram realizadas por André, Renato e Guilherme. A idealização e proposição do tema, análises e discussões teve direção principal do Prof. Dr. Mauricio Galo. Todos os autores contribuíram na redação e revisão do texto. 


\section{Referências}

ALBERS, B.; KADA, M.; WICHMANN, A. Automatic extraction and regularization of building outlines from airborne LiDAR point clouds. In: The International Archives of the Photogrammetry, Remote Sensing and Spatial Information Sciences. Prague, Czech Republic, v. XLI-B3, p. 555-560, 2016.

AVBELJ, J.; MÜLLER, R.; BAMLER, R. A metric for polygon comparison and building extraction evaluation. IEEE Geoscience and Remote Sensing Letters, v. 12, n. 1, 2015. p. 170-174.

AWRANGJEB, M. Using point cloud data to identify, trace, and regularize the outlines of buildings. International Journal of Remote Sensing, v. 37, n. 3, p. 551-579, 2016.

BRASIL. Constituição da República Federativa do Brasil de 1988. Promulgada em 5 de outubro de 1988.

BRASIL. Lei $\mathrm{n}^{\circ}$ 10.406, de 10 de janeiro de 2002. Institui o Código Civil. Brasilia, Diário Oficial da União, 10 de janeiro de 2002.

BRASIL. Lei $\mathrm{n}^{\circ}$ 10.257, de 10 de julho de 2001. Regulamenta os arts. 182 e 183 da Constituição Federal, estabelece diretrizes gerais da política urbana e dá outras providências. Brasilia, Diário Oficial da União, 10 de julho de 2001.

CARRILHO, A. C.; IVÁNOVÁ, I.; GALO, M. Quality assessment for automatic LiDAR data classification methods. In: XVIII SBSR - Simpósio Brasileiro de Sensoriamento Remoto, Santos - SP, 2017, pp. 6772-6779.

CARRILHO, A. C.; GALO, M. Extraction of building roof planes with stratified random sample consensus. The Photogrammetric Record, v. 33, p. 363-380, 2018 .

CARRIlHO, A. C.; GALO, M.; SANTOS, R. C.; PESSOA, G. G. Avaliação da extração automática de edificações a partir de dados lidar aerotransportado. In: Anais do COBRAC 2018, Florianópolis - SC, UFSC.

CENTENO, J. A. S.; DAROS, R.; GARGON, J. P. Extração de DTM e detecção de construções em áreas urbanas usando LiDAR. In: Anais do COBRAC 2016, Florianópolis - SC, UFSC.

DAL POZ, A.; HABIB, A. F.; MARCATO, V. J.; CORREIA, L. S. Uso de dados fotogramétricos no refinamento geométrico de contornos de telhados de 
edifícios extraídos de dados LASER. Boletim de Ciências Geodésicas, v. 15, n. 3, p. 594-614, 2009.

DAL POZ, A. P. Refinamento fotogramétrico de contornos de telhado extraídos de dados de varredura a LASER. Revista Brasileira de Cartografia, v. 66, n. 5, p. 1135-1144, 2014.

DSG - Diretoria de Serviço Geográfico. Especificação Técnica para a Aquisição de Dados Geoespaciais Vetoriais (ET-ADGV). 2 ed. Brasília: DSG, 2016.

EDELSBRUNNER, H.; KIRKPATRICK, D. G.; SEIDEL, S. On the shape of set of points in the plane. IEEE Transactions on Information Theory, v. IT-29, n. 4, p. 551-559, 1983.

FERREIRA, F. R.; LAROCCA, A. P. C.; CINTRA, J. P. Segmentação do espaço urbano por meio de dados LiDAR aerotransportado. Revista Brasileira de Cartografia, v. 67, n. 7, p. 1413-1420, 2015.

GALVANIN, E. A. S.; DAL POZ, A. P. Extraction of building roof contours from LiDAR data using a Markov-Random-Field-based approach. IEEE Transactions on Geoscience and Remote Sensing, v. 50, n. 3, 2012. p. 981-987.

GHAMISI, P.; HÖFLE, B.; ZHU, X. X. Hyperspectral and LiDAR data fusion using extinction profiles and deep convolutional neural network. IEEE Journal of Selected Topics in Applied Earth Observations and Remote Sensing. v. 10, n. 6, p. 3011-3024, 2016.

GONZÁLEZ-AGUILERA, D.; CRESPO-MATELLÁN, E.; HERNÁNDEZ-LÓPEZ, D.; RODRÍGUEZ-GONZÁLVEZ, P. Automated urban analysis based on LiDARderived building models. IEEE Transactions on Geoscience and Remote Sensing, v. 51, n. 3, 2013. p. 1844-1851.

JOCHEM, A.; HOFLE, B.; RUTZINGER, M.; PFEIFER, N. Automatic roof plane detection and analysis in airborne LiDAR point clouds for solar potential assessment. Sensors, v. 9, p5241-5262, 2009.

JUNG, J.; JWA, Y.; SOHN, G. Implicit regularization for reconstructing 3D building rooftop models using airborne LiDAR data. Sensors, v. 17, p. 27, 2017.

KANG, Z.; YANG, J.; ZHONG, R. A Bayesian-Network-Based classification method integrating airborne LiDAR data with optical images. IEEE Journal of 
Selected Topics in Applied Earth Observations and Remote Sensing, v. 10, n. 4, 1651-1661, 2017.

KIM, C.; HABIB, A. Object-based integration of photogrammetric and LiDAR data for automated generation of complex polyhedral building models. Sensors, v. 9, p. 5679-5701, 2009.

KWAK, E.; HABIB, A. Automatic representation and reconstruction of DBM from LiDAR data using Recursive Minimum Bounding Rectangle. ISPRS Journal of Photogrammetry and Remote Sensing, p. 171-191, 2014.

LAStools. Site: <http://rapidlasso.com/LAStools>, acessado em julho de 2018.

LEE, J.; HAN, S.; BYUN, Y.; KIM, Y. Extraction and regularization of various building boundaries with complex shapes utilizing distribution characteristics of airborne LiDAR points. ETRI Journal, v. 33, n. 4, p. 547-557, 2011.

MACHADO, Á. M. L. Extração automática de contornos de edificações utilizando imagem gerada por câmara digital de pequeno formato $e$ dados LIDAR. Tese de doutorado - Universidade Federal do Paraná (UFPR), Programa de Pós-Graduação em Ciências Geodésicas. Curitiba, p. 213. 2006.

MANNO-KOVACS, A.; SZIRANYI, T. Orientation-selective building detection in aerial images. ISPRS Journal of Photogrammetry and Remote Sensing, p. 94-112, 2015.

MAAS, H.; VOSSELMAN, G. Two algorithms for extracting building models from raw laser altimetry data. ISPRS Journal of Photogrammetry and Remote Sensing, v. 54, p. 153-163, 1999.

MENDES, T. S. G.; DAL POZ, A. P. Integração de imagem aérea de alta resolução e dados de varredura a laser na classificação de cenas urbanas para detectar regiões de via. Boletim de Ciências Geodésicas, v. 19, n. 2, p. 287-312, 2013.

OLIVEIRA, G. R. K. Uso integrado de dados lidar e imagens aéreas aplicado na extração de contornos de telhados de edificações. Dissertação de mestrado - Universidade do Estado de São Paulo (UNESP), Programa de PósGraduação em Ciências Cartográficas. Presidente Prudente, p. 105. 2016.

OLIVEIRA, G. R. K.; GALO, M. Extração de contornos de telhados de edificações através da combinação de dados LiDAR e imagens aéreas. Revista Brasileira de Cartografia, v. 70, n. 4, p. 1378-1408, 2018. 
RICHARDS, J. A. Remote sensing digital image analysis: an introduction. Londres: Springer, 2013. 494 p.

SAMPATH, A.; SHAN, J. Building boundary tracing and regularization from airborne LiDAR point clouds. In: International Archives of Photogrammetry and Remote Sensing, v. 73, p. 805-812, 2007.

SANTOS, R. C.; GALO, M.; CARRILHO, A. C. Building boundary extraction from LiDAR data using a local estimated parameter for alpha-shape algorithm. In: International Archives of Photogrammetry, Remote Sensing and Spatial Information Sciences, v. XLII-1, p. 127-132, 2018a.

SANTOS, R. C.; GALO, M.; CARRILHO, A. C.; PESSOA, G. G. Uso do algoritmo alpha shape na extração de contornos de edificações a partir de dados LiDAR. In: Anais do COBRAC 2018b, Florianópolis - SC, UFSC.

SANTOS, R. C.; PESSOA, G. G.; CARRILHO, A. C.; GALO, M. Building detection from lidar data using entropy and the k-means concept. In: International Archives of Photogrammetry, Remote Sensing and Spatial Information Sciences, v. XLII-2/W13, p. 969-974, 2019a.

SANTOS, R. C.; GALO, M.; CARRILHO, A. C. Extraction of building roof boundaries from LiDAR data using an adaptive alpha-shape algorithm. IEEE Geoscience and Remote Sensing Letters, 2019b.

SATARI, M. A Multi-resolution hybrid approach for building model reconstruction from LIDAR data. The Photogrammetric Record. v. 27, n. 139, p. 330-359, 2012.

SHEN, W. Building boundary extraction based on LiDAR point clouds data. In: The International Archives of the Photogrammetry, Remote Sensing and Spatial Information Sciences. Beijing, China, v. 37, p. 157-162, 2008.

SOKOLOVA, M.; JAPKOWICZ, N.; SZPAKOWICZ, S. Beyond accuracy, f-score and roc: a family of discriminant measures for performance evaluation. In: Proceedings of the AAAI'06 workshop on Evaluation Methods for Machine Learning. [S.l.: s.n.], 2006. p. 24-29.

TOMMASELLI, A. M. G.; GALO, M.; DOS REIS, T. T.; RUY, R.; MORAES, M. V. A.; MATRICARDI, W. V. Development and assessment of a data set containing frame images and dense airborne laser scanning point clouds. IEEE Geoscience and Remote Sensing Letters, v. 15, p. 192-196, 2018. 
WANG, J.; YANG, X.; QIN, X.; YE, X.; QIN, Q. An efficient approach for automatic rectangular building extraction from very high resolution optical satellite imagery. IEEE Transactions on Geoscience and Remote Sensing, v.12, n. 3, p. 487-491, 2015 .

YOUSEFHUSSIEN, M.; KELBE, D. J.; IENTILUCCI, E. J.; SALVAGGIO, C. A multi-scale fully convolutional network for semantic labeling of 3D point clouds. ISPRS Journal of Photogrammetry and Remote Sensing, [S.l.: s.n.], 2018, 14 p. 